\title{
Hipertrofia Ventricular Esquerda: Um Fenótipo, Duas Hipóteses, Três Lições
}

\author{
Left Ventricular Hypertrophy: One Phenotype, Two Hypotheses, Three Lessons
}

Patrícia Rodrigues, ${ }^{1}{ }^{\circledR 0}$ Ana Rita Soares, ${ }^{1}$ Ricardo Taipa, ${ }^{1}$ Sofia Ferreira, ${ }^{1}$ Hipólito Reis ${ }^{1}$

Centro Hospitalar Universitário do Porto EPE, ${ }^{1}$ Porto - Portugal

\section{Histórico médico}

Uma mulher de 58 anos com polineuropatia amiloidótica familiar causada pela mutação Val30Met (p.Val50Met) no gene da transtirretina (TTR) começou a apresentar sintomas neuropáticos aos 37 anos e uma biópsia da glândula salivar confirmou a deposição de amiloide TTR. Foi submetida a transplante hepático sete anos após o início dos sintomas e fazia uso de imunossupressores, com alterações neurológicas estáveis desde então. Ela também apresentava insuficiência renal crônica (estágio 3b) e um marcapasso implantado devido a doença do nó sinusal.

\section{História da apresentação}

A paciente foi encaminhada ao Ambulatório de Cardiologia 14 anos após o transplante hepático, por apresentar dispneia progressiva e edema nos dois pés. Ao exame físico, apresentava sinais de congestão periférica e pulmonar.

Os resultados do ECG e a interrogação do marcapasso revelaram fibrilação atrial e estimulação ventricular com frequência cardíaca controlada.

O ecocardiograma transtorácico revelou hipertrofia ventricular esquerda (HVE) importante, função sistólica preservada e disfunção diastólica - Figura 1.

Iniciou anticoagulação oral e diuréticos, com melhora clínica.

\section{Diagnóstico diferencial}

Na presença de insuficiência cardíaca com HVE, devemos primeiro considerar as condições de carga, como hipertensão ou doença valvar, que não foram observadas nesta paciente.

A cardiomiopatia hipertrófica $(\mathrm{CMH})$ sarcomérica era um diagnóstico possível, que pode se apresentar com diferentes padrões de HVE, sendo a causa genética mais comum de HVE. A doença de Fabry poderia ser outra possibilidade, embora mais rara.

\section{Palavras-chave}

Hipertrofia Ventricular Esquerda; Insuficiência Cardíaca; Cardiomiopatia Hipertrófica; Amiloidose; Neuropatias Amiloides Familiares/diagnóstico por imagem.

Correspondência: Patrícia Rodrigues •

Centro Hospitalar Universitário do Porto EPE - Largo Professor Abel Salazar Porto 4099-001 - Portugal

E-mail:pfdrodrigues@gmail.com

Artigo recebido em 09/02/2021, revisado em 19/04/2021, aceito em 09/06/2021

DOI: https://doi.org/10.36660/abc.20210103
Contudo, nesta paciente com uma mutação conhecida, o diagnóstico mais provável era Cardiomiopatia Amiloidótica por Transtirretina (CM-ATTR). Pacientes sem cardiomiopatia significativa no momento do transplante de fígado, particularmente se sua mutação não era Val30Met, podem progredir depois, devido à deposição aumentada de proteína do tipo selvagem. ${ }^{1}$

\section{Investigações}

Surpreendentemente, a cintilografia com Tecnécio-99m (TC-99m) com ácido 3,3-difosfono-1,2-propanodicarboxílico (DPD) foi negativa (escore zero de Perugini) - Figura 2.

A amiloidose AL foi excluída após análise de imunofixação (urina de $24 \mathrm{~h}$ e soro) e cadeias leves livres no soro.

A ressonância magnética cardiovascular (RMC) não foi realizada, uma vez que os eletrodos e gerador do marcapasso não eram condicionais para a RMC e a paciente era claustrofóbica. Foi solicitada biópsia endomiocárdica, que foi negativa para amiloide e não mostrou alterações significativas. Nesse ponto, outros diagnósticos foram reconsiderados para HVE.

Um estudo genético com um painel de $\mathrm{CMH}$ (incluindo doença de Fabry) foi solicitado e uma variante provavelmente patogênica em heterozigose foi encontrada no gene MYH7 (p.Arg783Leu). Isso nos fez questionar se o fenótipo poderia ser atribuído à $\mathrm{CMH}$.

No entanto, a paciente necessitava de altas doses de diuréticos (pelo menos $120 \mathrm{mg}$ de furosemida por dia para permanecer euvolêmica), embora não houvesse obstrução da via de saída do ventrículo esquerdo e era dependente de marcapasso. Revisando o ecocardiograma (Figura 1), ela apresentava um padrão de fluxo transmitral restritivo, baixas velocidades da onda S' no Doppler tecidual e ligeiro derrame pericárdico. Todos esses achados não são típicos de $\mathrm{CMH}$.

Foi solicitada revisão da biópsia endomiocárdica por um patologista mais experiente, a qual na verdade mostrou infiltração amiloide grave (Figura 3).

\section{Discussão}

Faltam critérios universalmente aceitos para o diagnóstico de cardiomiopatia amiloide, especificamente para CM-ATTR, e o algoritmo proposto por Gillmore et al. ${ }^{2}$ ajuda a determinar o tipo de amiloidose, mas parte de achados "sugestivos de amiloidose cardíaca", que são bastante amplos. Uma recente declaração de posição europeia propõe um algoritmo mais claro para a suspeita e diagnóstico de amiloidose cardíaca. ${ }^{3}$

Geralmente, o diagnóstico requer aumento da espessura da parede ventricular (usualmente $>12 \mathrm{~mm}$ ), combinado com os resultados de exames hematológicos, cintilografia óssea e, às vezes, biópsia. 


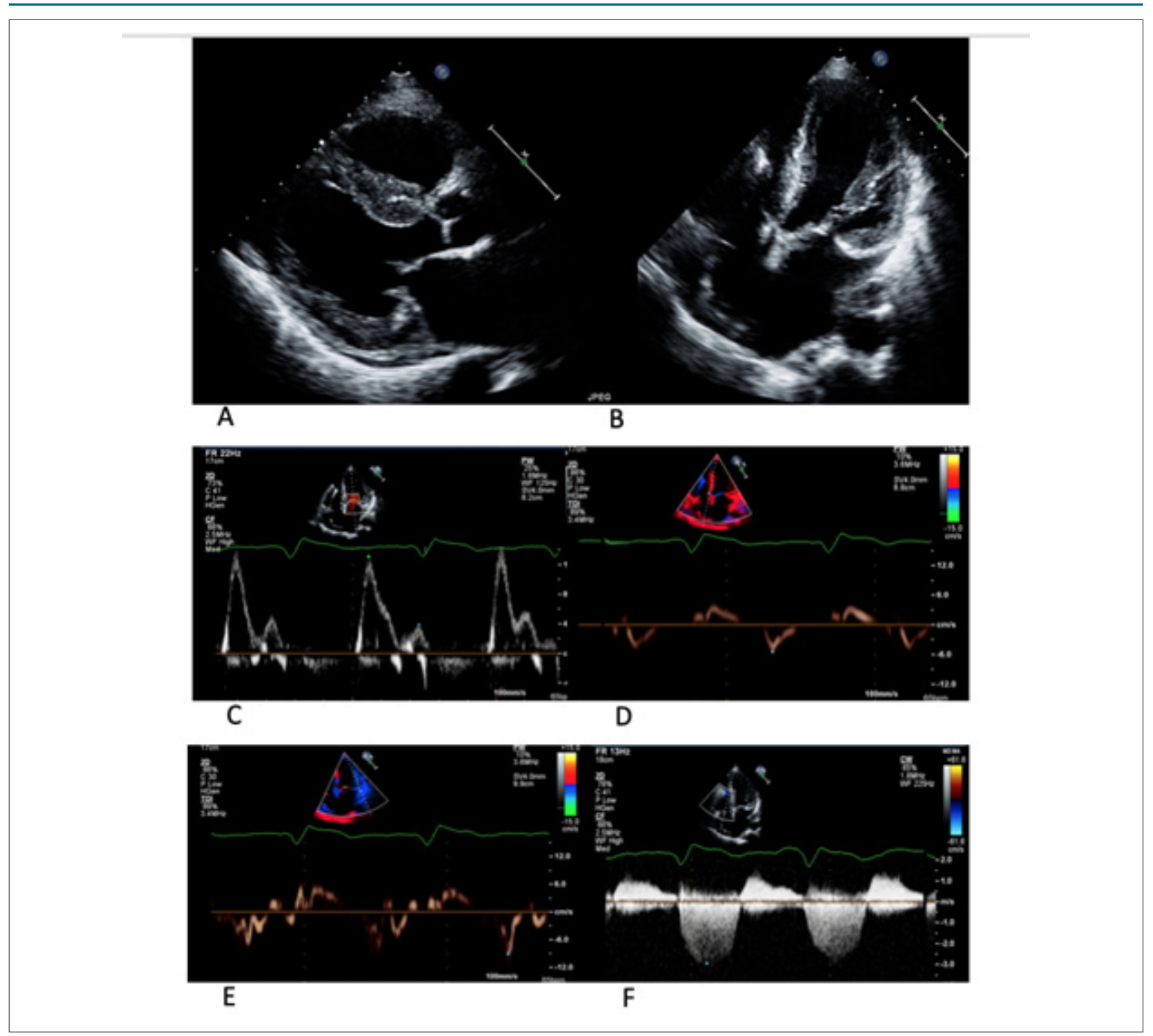

Figura 1 - Achados da ecocardiografia transtorácica. Os painéis $A$ (projeção paraesternal de eixo longo) e $B$ (apical de 4 câmaras) mostram aumento da espessura da parede (máximo de $15 \mathrm{~mm}$ no septo interventricular basal) e átrio esquerdo levemente dilatado (volume indexado pela superfície corporal de $40 \mathrm{~mL} / \mathrm{m}^{2}$ ). 0 painel C mostra uma razão E/A aumentada de 3,4. 0 painel D mostra uma velocidade de e' lateral de $9 \mathrm{~cm} / \mathrm{s}$ e o painel E uma e' septal de $5 \mathrm{~cm} / \mathrm{s}$, dando uma média E/e' de 15. No painel F, a velocidade de regurgitação tricúspide é estimada em $2,9 \mathrm{~m} / \mathrm{s}$ com Cw Doppler. Portanto, a paciente preenchia os critérios para disfunção diastólica.

A cintilografia com ${ }^{99 m} \mathrm{Tc}$-DPD mostrou excelente sensibilidade e especificidade para detectar CM-ATTR, muitas vezes dispensando a confirmação histológica, ${ }^{2}$ particularmente quando um escore de Perugini de 2 ou 3 (captação cardíaca moderada ou intensa) é observado. ${ }^{4,5}$ No entanto, mais recentemente, achados falso-negativos em imagens de radionuclídeos foram encontrados em pacientes com a mutação TTR Val30Met e início precoce de sintomas neurológicos. ${ }^{6} \mathrm{~A}$ causa parece estar relacionada ao fato de que esses pacientes apresentam exclusivamente fibrilas do tipo B (comprimento total), com baixa avidez por ${ }^{99 \mathrm{~m}} \mathrm{Tc}-\mathrm{DPD}$, ao contrário de pacientes com início tardio ou outras mutações, que também têm fibrilas do tipo A (truncadas). ${ }^{7}$ Nos primeiros casos, uma investigação adicional, incluindo biópsia endomiocárdica, pode ser necessária.
Curiosamente, nesta paciente, a biópsia endomiocárdica foi inicialmente negativa, levando-nos a explorar outros diagnósticos, a saber, $\mathrm{CMH}$ (como as biópsias eram muito pequenas e provenientes do ventrículo direito, a hipertrofia dos cardiomiócitos pode passar despercebida). Entretanto, devemos reconhecer que um patologista com experiência no diagnóstico de amiloidose é crucial.

Nosso grupo e vários outros descreveram o desenvolvimento de CM-ATTR anos após o transplante de fígado, não apenas em pacientes com início tardio ou mutações não-Val30Met, como relatado inicialmente, mas também em pacientes com Val30Met de início precoce. Esse fenômeno foi atribuído a mecanismos de semeadura: pequenos depósitos de fibrilas amiloides com um precursor de TTR mutado podem promover o acúmulo 


\section{Carta Científica}

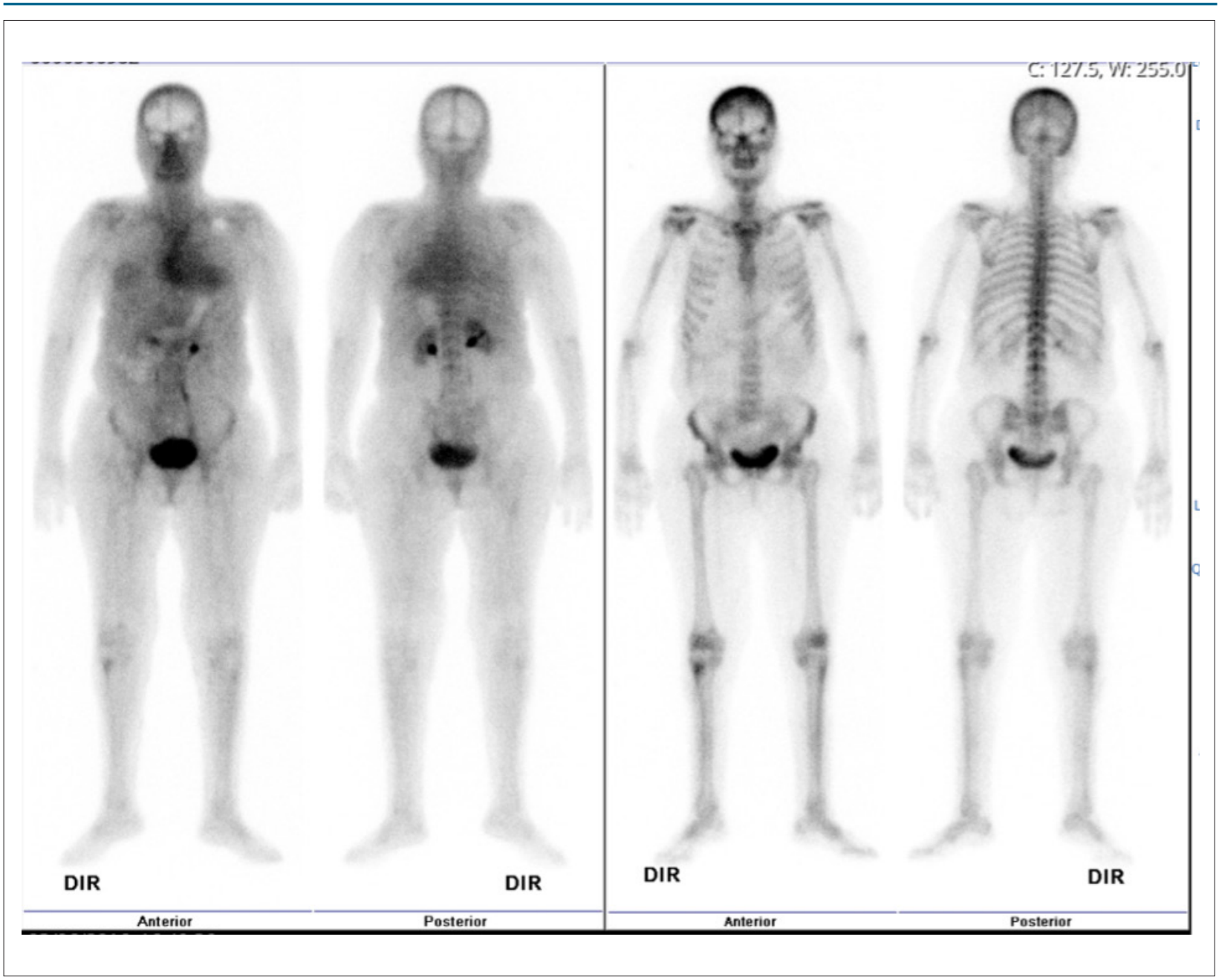

Figura 2 - Imagens de cintilografia com Tecnécio-99m (Tc-99m) com DPD (99m Tc-DPD). As imagens à esquerda foram obtidas 10 minutos após a administração do ${ }^{99 m}$ Tc-DPD e as imagens à direita 2 horas depois. O escore de Perugini foi zero, o que significa ausência de captação cardiaca e captação óssea normal.

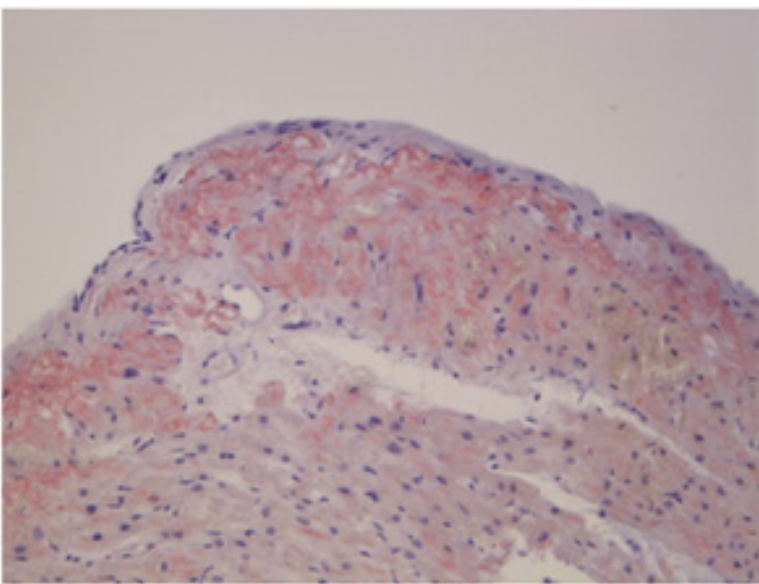

A

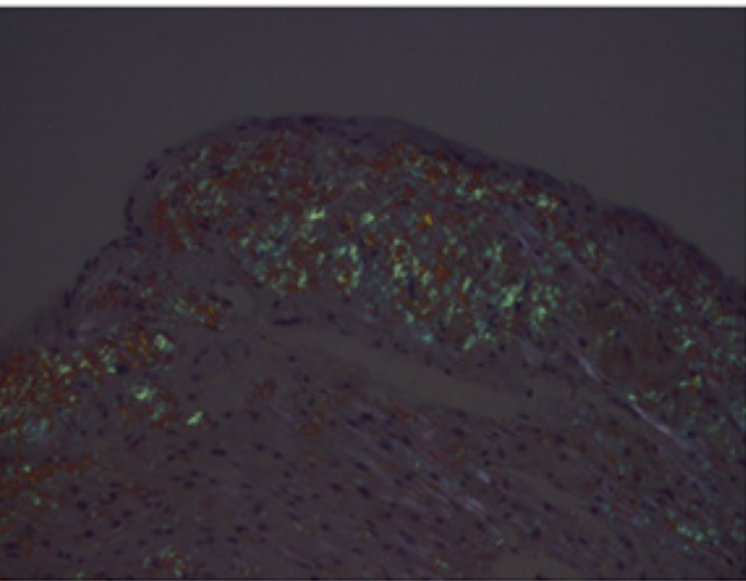

B

Figura 3 - Biópsia endomiocárdica, mostrando infiltração amiloide no interstício miocárdico e particularmente no endocárdio. Coloração com Vermelho Congo (A) e coloração com Vermelho Congo sob luz polarizada (B); amplificação 200x. 
tardio de fibrilas de tipo selvagem. No entanto, ainda não entendemos por que esses pacientes não têm um escore positivo na cintilografia com ${ }^{99 \mathrm{~m} T c-D P D}$ com mais frequência, similar a pacientes com doença do tipo selvagem. Por fim, o teste genético tem sido cada vez mais útil na investigação das cardiomiopatias, mas os resultados precisam ser discutidos com cautela, pois podem ter implicações no diagnóstico e no rastreamento familiar. $\mathrm{O}$ conhecimento acumulado sobre cardiogênese vai esclarecer a classificação de algumas variantes. Quando uma variante patogênica ou provavelmente patogênica é encontrada, o rastreamento genético é geralmente oferecido aos membros da família.

\section{Conclusões}

Nosso diagnóstico final foi CM-ATTR, embora os primeiros exames parecessem ter afastado essa hipótese, destacando o fato de que as biópsias endomiocárdicas são altamente dependentes do patologista e a cintilografia com ${ }^{99 \mathrm{~m}} \mathrm{Tc}-\mathrm{DPD}$ pode apresentar resultados falso-negativos. Além disso, os resultados dos testes genéticos em $\mathrm{CMH}$ precisam ser interpretados no contexto clínico, uma vez que o achado de uma mutação, principalmente se ela não for claramente patogênica, não significa que a mesma seja a causadora do fenótipo.

Infelizmente, atualmente não há medicamentos aprovados para o tratamento da cardiomiopatia amiloide em pacientes transplantados; contudo, esperamos que isso mude em um futuro próximo.

\section{0 que já se sabe sobre esse assunto? 0 que este estudo adiciona?}

Este caso fornece três lições importantes:

- a identificação da causa da HVE é frequentemente negligenciada, mas buscar hipóteses diferentes e identificar a etiologia tem implicações clínicas para o paciente e sua família;

\section{Referências}

1. Stangou AJ, Hawkins PN, Heaton ND, Rela M, Monaghan M, Nihoyannopoulos $\mathrm{P}$ et al. Progressive cardiac amyloidosis following liver transplantation for familial amyloid polyneuropathy: implications for amyloid fibrillogenesis. Transplantation. 1998; 66(2): 229-33. doi: 10.1097/00007890-199807270-00016.

2. Gillmore JD, Maurer MS, Falk RH, Merlini G, Damy T, Dispenzieri A et al. Nonbiopsy Diagnosis of Cardiac Transthyretin Amyloidosis. Circulation. 2016; 133(24): 2404-12. doi:10.1161/CIRulationAHA.116.021612.

3. Garcia-Pavia P, Rapezzi C, Adler Y, Arad M, Basso C, Brucato A et al. Diagnosis and treatment of cardiac amyloidosis: a position statement of the ESC Working Group on Myocardial and Pericardial Diseases. Eur Heart J. 2021;42(16):1554-68. doi:10.1093/eurheartj/ehab072

4. Perugini E, Guidalotti PL, Salvi F, Cooke RM, Pettinato C, Riva L etal. Noninvasive etiologic diagnosis of cardiac amyloidosis using 99mTc-3,3-diphosphono-1,2-
- devemos estar cientes das armadilhas da identificação de amiloide em biópsias, particularmente a importância de um patologista experiente;

- A cintilografia com ${ }^{99 m}$ Tc-DPD também tem limitações, principalmente em pacientes com mutação Val30Met de início precoce; combinar a história clínica com os resultados de diferentes exames é fundamental para o diagnóstico da amiloidose cardíaca.

\section{Contribuição dos autores}

Concepção e desenho da pesquisa e Obtenção de dados: Rodrigues P; Análise e interpretação dos dados e Redação do manuscrito: Rodrigues P, Soares AR, Taipa R; Revisão crítica do manuscrito quanto ao conteúdo intelectual importante: Rodrigues P, Soares AR, Taipa R, Ferreira S, Reis H.

\section{Potencial conflito de interesse}

Não há conflito com o presente artigo

\section{Fontes de financiamento}

O presente estudo não teve fontes de financiamento externas.

\section{Vinculação acadêmica}

Não há vinculação deste estudo a programas de pósgraduação.

\section{Aprovação ética e consentimento informado}

Este estudo foi aprovado pelo Comitê de Ética do Centro Hospitalar do Porto sob o número de protocolo 2017.219 (189-DEFI/181-CES). Todos os procedimentos envolvidos nesse estudo estão de acordo com a Declaração de Helsinki de 1975 , atualizada em 2013. O consentimento informado foi obtido de todos os participantes incluídos no estudo. propanodicarboxylic acid scintigraphy. J Am Coll Cardiol. 2005; 46(6): 1076-84. doi: 10.1016/j.jacc.2005.05.073.

5. Dorbala S, Ando Y, Bokhari S, Dispenzieri A, Falk RH, Ferrari VA, et al ASNC/ AHA/ ASE/ EANM/ HFSA/ ISA/ SCMR/ SNMMI Expert Consensus Recommendations for Multimodality Imaging in Cardiac Amyloidosis. J Card Fail .2019; 25(11): e1-39. doi:10.1016/j.cardfail.2019.08.001.

6. Coutinho MCA, Cortez-Dias N, Cantinho G, Gonçalves S, Menezes MN, Guimarães T et al. The sensitivity of DPD scintigraphy to detect transthyretin cardiac amyloidosis in V30M mutation depends on the phenotypic expression of the disease. Amyloid. 2020; 27(3):174-83. doi:10.1080/13506129.2020.1 744553.

7. Pilebro B, Suhr OB, Näslund U, Westermark P, Lindqvist P, Sundström T. $(99 \mathrm{~m}) \mathrm{Tc}-\mathrm{DPD}$ uptake reflects amyloid fibril composition in hereditary transthyretin amyloidosis. Ups J Med Sci 2016; 121(1):17-24. doi: 10.3109/03009734.2015.1122687. 